\title{
Research on Key Technology of Matching for Bulldozer Radiator Cloud Design Platform
}

\author{
Wang $\mathbf{J i}^{1, \mathrm{a}}$, Zhang Ruwei ${ }^{2, \mathrm{~b}}$, Tian Congfeng ${ }^{3, \mathrm{c}}$, Yan Wei ${ }^{4, \mathrm{~d}}$
}

1School of Energy \& Power Engineering, Shan Dong University, Ji Nan, China, 250061

2Jining Coordination Information Technology Co. Ltd, Ji Ning, China, 272073

3Jining Coordination Information Technology Co. Ltd, Ji Ning, China, 272073

4School of Energy \& Power Engineering, Shan Dong University, Ji Nan, China, 250061

a949182042@qq.com, bzhang_rw01@163.com, ctiancongfeng@163.com dyw65986092@sina.com

Key words: Bulldozer radiator; Cloud design platform; Cloud matching

\begin{abstract}
In view of the urgent need of radiator matching during the process of modern bulldozer design and considering the diversity as well as the individualization of different bulldozer types, a cloud design platform for bulldozer radiator which integrates cloud computing, parameterized and modular rapid matching is proposed. First, the cloud matching demand and characteristics of bulldozer radiator are analyzed. Then the general framework of bulldozer radiator cloud design platform is constructed, and the architecture as well as service mode of the cloud design platform are analyzed. Then the core technology of the cloud matching system is described. The feasibility of this cloud-based design platform is verified by the model of a bulldozer: The platform can get an optimal match quickly and accurately, and can also output detailed radiator parameters.
\end{abstract}

\section{Introduction}

As an important part of construction machinery, the bulldozer is an indispensable "driving force" in China's manufacturing industry. Since electronic control diesel engines have been widely used as the driving force on the bulldozers nowadays [1], the requirements of corresponding bulldozer radiator are also more and more strict.

However, there are a wide variety of bulldozers on the market. And the radiators required for different types of bulldozers are also different. During the research and development process of the bulldozer, the radiator often needs a targeted production, and even needs to be made to order. Meanwhile, there are lots of domestic radiator manufacturers and also lots of types of radiators. The problems of product singularity and weak pertinence are very serious.

$\mathrm{Li}$ Bohu and his team put forward the idea of cloud manufacturing for the first time in June 2009 [2]. As a manufacturing service platform based on network and cloud, the cloud manufacturing service platform is a new network manufacturing model, which is based on users' needs to organize online manufacturing resources, and to provide them with a variety of on-demand manufacturing services. It emphasizes the idea that "centralized use of decentralized resources, and centralized resources for decentralized service", which is an effective way to solve the problems above.

Based on the analysis above, the design resources of the bulldozer manufacturers and radiator manufacturers are deployed to the cloud design platform and integrated with the system to solve the problem such as uneven resource distribution, resource sharing, lack of innovation ability and poor cooperation between enterprises and to realize the management and sharing of design resources. 


\section{Needs and characteristics of cloud matching on bulldozer radiator}

The development of the bulldozer is a systematized and complex process. In order to match the bulldozer and radiator quickly and efficiently, cloud manufacturing service needs to have the following characteristics in addition to those of general SME cloud manufacturing services [3]:

(1)Convenience of business collaboration. Bulldozer radiator matching is a complex process, including existing product matching and new product design matching. The process needs the collaboration between enterprises of strong professional and advanced R\&D capability in different fields. The collaboration between the enterprises is close and complex.

(2) Accuracy of resource description. In view of the diversity of products, various access standards of bulldozer manufacturers and the customization requirements of many overseas enterprises, new product development cloud manufacturing services must have large-scale resources, and resource properties change frequently.

(3) Efficiency of resource search. Cloud manufacturing is to realize optimal allocation of a nationwide manufacturing resources as well as resource sharing in different places. But the bulldozer radiator industry is mostly regional manufacturing cluster industry [4]. Therefore, the platform needs to provide an efficient search mechanism for the individual needs of the resource demand side.

\section{The Architecture of Bulldozer Radiator Cloud design platform}

Bulldozer radiator matching generally includes sample matching, simulation analysis, sample trial, test analysis, collaborative analysis, performance evaluation and other major processes. The development and operation service mode is shown in the figure below.

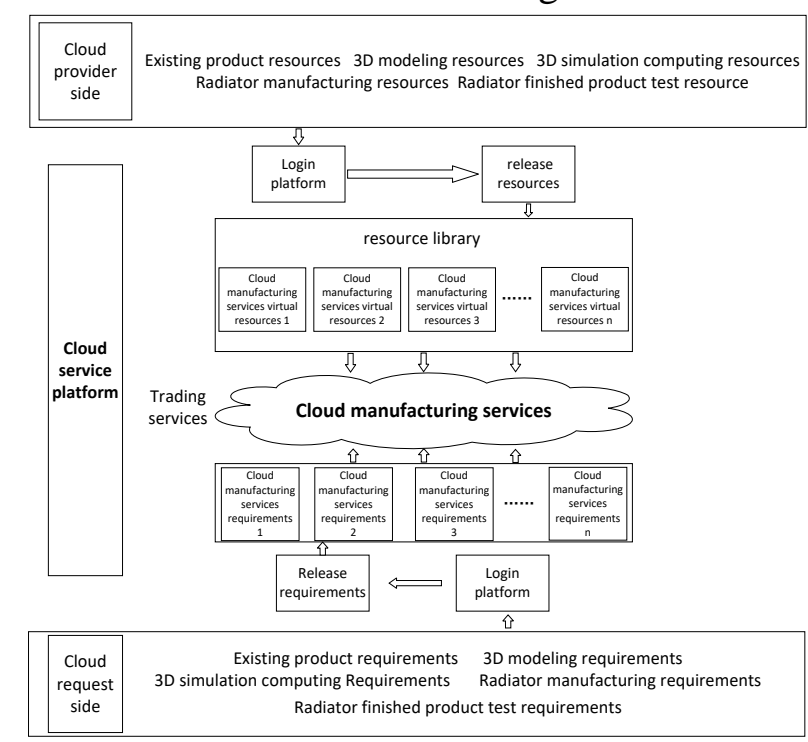

Figure1 Architecture of Radiator Cloud design platform

First, both of the demanding side and providing side have to sign in the platform, the demanding side uses the platform demand release module to describe and release the demand of the new product. Meanwhile, the providing side uses the resource registration and publish module of the platform to register and publish the product research and development service resources.

Then, the demanding side searches through the platform matching combination module to obtain the resources related to the new product development service of the radiator and makes the optimal selection as well as application of the resources. The providing side uses the platform matching 
combination module to receive and confirm the resource application request from the demanding side. This means the demanding side and providing side establish a formal partnership.

After the transaction relationship is determined, the demanding side and providing side can realize the modeling and deployment configuration of the new product development service business process of the radiator through the service business management module. Each service provider cooperates in accordance with the business process. Through the visual monitoring function provided by service business management module, visual monitoring of the service business is realized.

After the service business is completed, the demanding side can pay on demand using the service transaction management module of the platform to achieve the management and monitoring of entire transaction process. And payment through a third-party payment platform is also supported.

The knowledge generated during the transaction process and the knowledge of the new product development that can be shared can realize gathering and sharing through the knowledge gathering and service networked community module. The module also supports the data mining and statistical analysis of the new radiator development data.

\section{Core technology of cloud platform intelligent matching}

The cloud matching design of radiators is introducing the intelligent matching method into the design of radiator, in order to shorten the design cycle and improve the design efficiency.

The core technology of intelligent matching is case-based reasoning (CBR) technology [5]. CBR technology uses the K-Nearest Neighbor method to search the case library to match examples. Euclidean distance is usually used to measure the similarity in the nearest neighbor search method. The nearest neighbor search method calculates the similarity between the target instance and the source instance by using the Euclidean distance. Then the system will recommend some examples of higher similarity to the designers for reference and reuse.

When the feature is identified, the Euler distance between the features should be calculated to calculate the similarity between them, and then the overall similarity between the two instances should be calculated. The results of high similarity will be recommended to the designers and the system will give some suggestions for changes.

\section{An example of Intelligent Cloud Matching for Bulldozer Radiator}

We have built a Windows Form platform, using Visual Studio 2010 as a development environment and $\mathrm{C \#}$ as a language. We take a bulldozer made in China as an example to demonstrate the intelligent matching of bulldozer radiators

Bulldozer features include model traction、dozer form、 Engine rated power、 heat dissipation、 cooling style、 installation dimensions of radiator (length * width * height) etc.

Radiator features include model 、material 、core structure size $(\mathrm{l} * \mathrm{w} * \mathrm{~h})$ 、 water pipe size (long axis * short axis)、 water pipe lines and so on a number of characteristic parameters.

In order to ensure the standardization and accuracy of system programming, the feature parameters are set uniformly, which are as follows in this table. 
Table1 radiator features list

\begin{tabular}{llll}
\hline Feature item & Eigenvalues & Feature item & Eigenvalues \\
\hline power ratings & R_Pow & maximum power & P_max \\
maximum drawing force & T_max & heat dissipation & $Q_{W}$ \\
radiator name & R_Nam & heat dissipation type & R_Typ \\
radiator material & R_Met & number of water pipes & P_Row \\
radiator number & R_Num & water pipe number & P_Num \\
\hline
\end{tabular}

The required feature items are selected from the above features, then they are imported into the system and matched.

Case database maintains the relevant structure and function parameters of the radiator supplied with the bulldozer engine products which are currently collected. Figure 2(a) shows a case database of an intelligent matching bulldozers with radiators. Enter the selected feature items and eigenvalues, then set their weight, as shown in Figure 2(b). Click start to produce the best result of the match and generate the report, as shown in Figure 2(c)

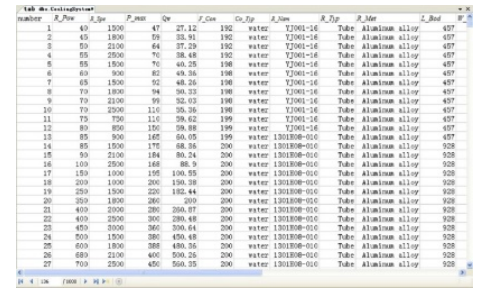

(a)

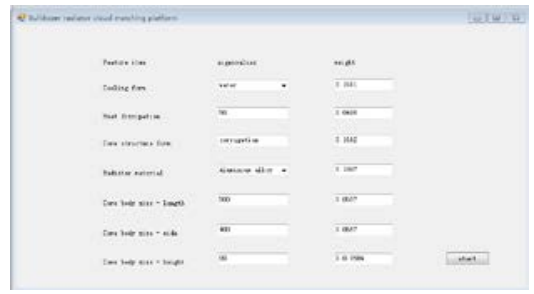

(b)

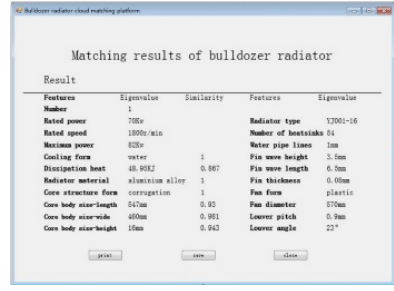

(c)

Figure2 Operation interface

\section{Conclusion}

Aiming at the disadvantages of the matching process of bulldozer radiator, such as uneven distribution of resources、 high design cost、 low efficiency, a cloud design platform for bulldozer radiator has been developed based on cloud design technology. This paper studies the design process, the design framework and the service mode of the cloud service platform deeply and construct the intelligent matching function framework of bulldozer radiator. Finally, we take a typical bulldozer radiator matching as an example to verify the feasibility of the platform.

\section{Acknowledgements}

The research was supported by China Torch Program under Grant No. $2015 G H 720903$.

\section{Reference}

[1]Li Xiangyang CFD Analysis and Optimization on Underhood Cooling System of SD16YE Bollduzer. [D].Shandong University, 2015.

[2]Bohu Li, Lin Zhang, Shilong Wang, Fei Tao, Junwei Cao, Xiaodan Jiang, Xiao Song, Xudong Chai. Cloud manufacturing: A new service-oriented networked manufacturing model. [J]. computer integrated manufacturing system, 2010, (01): 1-7+16.

[3]Yin Chao, Huang Biqing, Liu Fei, Wen Lijie, Wang Zhaokun, Li Xiaodong, Yang Shuping, Ye Dan, Liu Xianhui.Common key technology system of cloud manufacturing service platform for 
small and medium enterprises. [J]. computer integrated manufacturing system, 2011, (03): 495-503.

[4]Yin Hankun, Yin Chao, Gong Xiaorong,, Wang Mingyuan. Cloud manufacturing services platform general framework and key technologies for automobile and motorcycle parts new product development. [J]. computer integrated manufacturing system, 2013, (09): 2332-2339.

[5] Jian Qiu.Study on Intelligent Design of Automotive Radiator Based on Hybrid Case-based Reasoning. [D].Shandong University, 2014. 